\section{Aus Fehlern lernen}

D en Herausgebern dieses Buches gebührt großer Respekt dafür, konzeptionelles Neuland betreten zu haben und nicht therapeutische Erfolge, sondern gerade mögliche Fehlerquellen und Misserfolge offen zu benennen und zu diskutieren. Nur so kann es gelingen, aus Fehlern zu lernen und zukünftigen Schaden von den Patienten abzuwenden.

Auf über 300 Seiten stellen namhafte nationale Experten in 19 Kapiteln kurz die Grundlagen, im Wesentlichen aber vor allem die praktischen Vorgehensweisen und die hierbei zu beachtenden Fallstricke und Fehlerquellen dar. Dabei fließen immer wieder publizierte wie auch persönliche Erfahrungen in die Bewertung ein. Besprochen werden Therapien der wichtigsten Dermatosen, aber beispielsweise auch das richtige diagnostische Vorgehen in den Bereichen Mykologie und Bakteriologie. Ausführlich wird der kosmetische Bereich einschließlich der Lasertherapie und der Anwendung von Botulinumtoxin $A$ angesprochen.

Das Buch besticht nicht nur durch die ausgesprochen praxisorientierte Darstellung, sondern auch durch die überaus reiche Bebilderung und die didaktisch sinnvolle Einbindung von Tabellen und Abbildungen. Bisweilen fiel offensichtlich die Abgrenzung der hier intendierten Inhalte von dem grundlegenden Lehrbuchwissen nicht immer leicht. Insgesamt stellt das Buch von Köhn und Ring einen ganz wesentlichen und bislang einmaligen Beitrag zur Qualitätssicherung dar und sollte in der Hand des praktisch tätigen Dermatologen nicht fehlen.

Prof. Dr. Torsten Schäfer, Lübeck

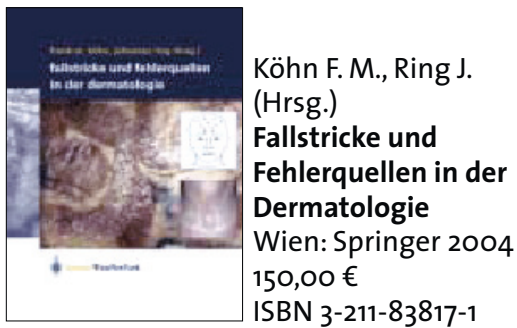

\title{
Dermatologie für alle
}

$\mathrm{H}$ autkrankheiten erkennen, diagnostizieren und behandeln - mithilfe dieses praxisrelevanten und benutzerfreundlichen Fotoatlasses von Konrad Bork und Wolfgang Bräuninger, der nun bereits in der dritten Auflage erscheint, wird dies deutlich erleichtert. Auch dem Nicht-Dermatologen erschließen sich zahlreiche interessante Aspekte dieses faszinierenden Fachgebietes. Das macht das vorgestellte Buch gerade auch für Allgemeinärzte, Internisten sowie Kinder- und HNO-Ärzte zu einem überaus interessanten Nachschlagewerk. Klinische Merkmale werden hervorgehoben, wenn sie für die diagnostische Interpretation der Symptome notwendig sind. Gegenüber der zweiten Auflage wurde weiteres erstklassiges Bildmaterial eingefügt, praxisrelevante differenzialdiagnostische Erwä- gungen - insbesondere in den Kapiteln „Arzneimittelnebenwirkungen“, „Urtikaria“ und „Angioödem" - wurden ergänzt.

Alle Leser werden von Konzept und Ausführung dieses Buches begeistert sein. Die klare didaktische Struktur verschafft einen raschen Überblick über die wichtigsten Hautkrankheiten mit wertvollem Praxisbezug.

Prof. Dr. Ludger Klimek, Wiesbaden

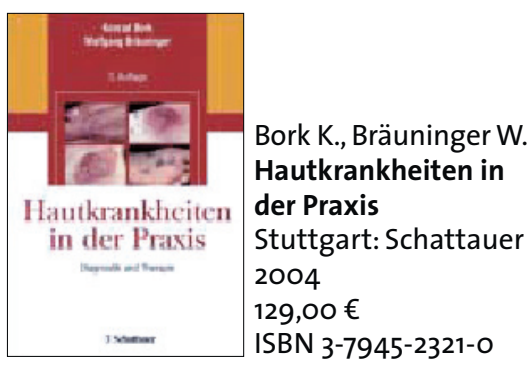

\section{Allergieprävention weltweit}

A uf rund 200 Seiten stellen die Herausgeber dieses Buches das Ergebnis eines gemeinsames Projekts von World Allergy Organization (WAO) und $\mathrm{WHO}$ vor. Dabei werden vor allem Aspekte der Primärprävention von Allergien und Asthma behandelt, die Sekundär- und Tertiärprävention aber ebenfalls angesprochen. Eine Vielzahl von namhaften internationalen Experten hat dazu den aktuellen Wissensstand zusammengetragen und bewertet. Die abgehandelten Themen betreffen die Genetik, Umwelteinflüsse, immunologische Einflüsse und die Vorhersage bzw. Frühdiagnose. Im Bereich der Präventionsmaßnahmen wird zwischen Frühinterventionen und dem Bereich der berufsbedingten Allergien unterschieden. Zur Sprache kommen ebenfalls Schulungsprogramme und Krankheitskosten sowie die potenziell positiven Auswirkungen von Präventionsmaßnahmen auf die Krankheitskosten. In der Zusammenfassung werden die konkreten Präventionsempfehlungen dargestellt, die um laiengerechte Hand- lungsempfehlungen und Merkblätter ergänzt werden.

Formal methodisch betrachtet wird nicht immer deutlich, ob den einzelnen Kapiteln eine systematische Literatursuche zugrunde liegt und ob für die Verabschiedung der Empfehlungen ein formales Konsensusverfahren gewählt wurde. Der Text wird durch nur wenige Tabellen und Abbildungen aufgelockert. Insgesamt stellt das Buch nicht nur für an Prävention Interessierte eine sehr empfehlenswerte und hochaktuelle Lektüre dar, das für wesentliche Bereiche der Allergologie den Wissensstand kompetent zusammenfasst.

Prof. Dr. Torsten Schäfer, Lübeck

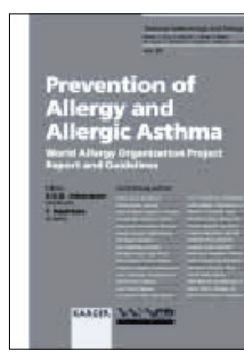

Johansson S.G.O., Haahtela T. (ed.) Prevention of Allergy and Allergic Asthma. World Allergy Organization Project Report and Guidelines Basel: Karger 2004 $127,00 €$ ISBN 3-8055-7810-5 